\title{
Causative allergen was confirmed by gas chromatograph mass spectrometer (GC/MS) in a patient with severe contact dermatitis due to goalkeeper gloves
}

\author{
Atsuko Aizawa ${ }^{1}$, Akiko Ito ${ }^{1}$, Yukiko Masui ${ }^{1}$, Kazumi Sasaki ${ }^{2}$, Mitsuru Numata ${ }^{2}$, Yutaka Ishimura ${ }^{2}$ and Riichiro Abe \\ 1 Division of Dermatology, Niigata University Graduate School of Medicine and Dental Science, Niigata, Japan \\ 2 National Institute of Technology and Evaluation, Tokyo, Japan
}

\section{Introduction:}

Daily life manufactures are required to disclose only raw materials, making it difficult to identify any ingredients that cause allergic reactions. To identify the causative allergen, National Institute of Technology and Evaluation (NITE) in Japan investigates the ingredients by gas chromatography mass spectrometry (GC/MS). In the present study, we used patch testing (PT) and GC/MS to identify the cause of severe contact dermatitis by the use of goalkeeper gloves.

\section{Patient:}

A 16-year-old male presented with a 2year history of pruritic erythema and fissures on the palms, dorsal hands and wrists. He had been a goalkeeper in a soccer team for 5 years. Application of steroid ointment had not been effective. As his skin lesions were confined to an area of skin covered by his goalkeeper gloves, we suspected that these gloves had triggered contact dermatitis.

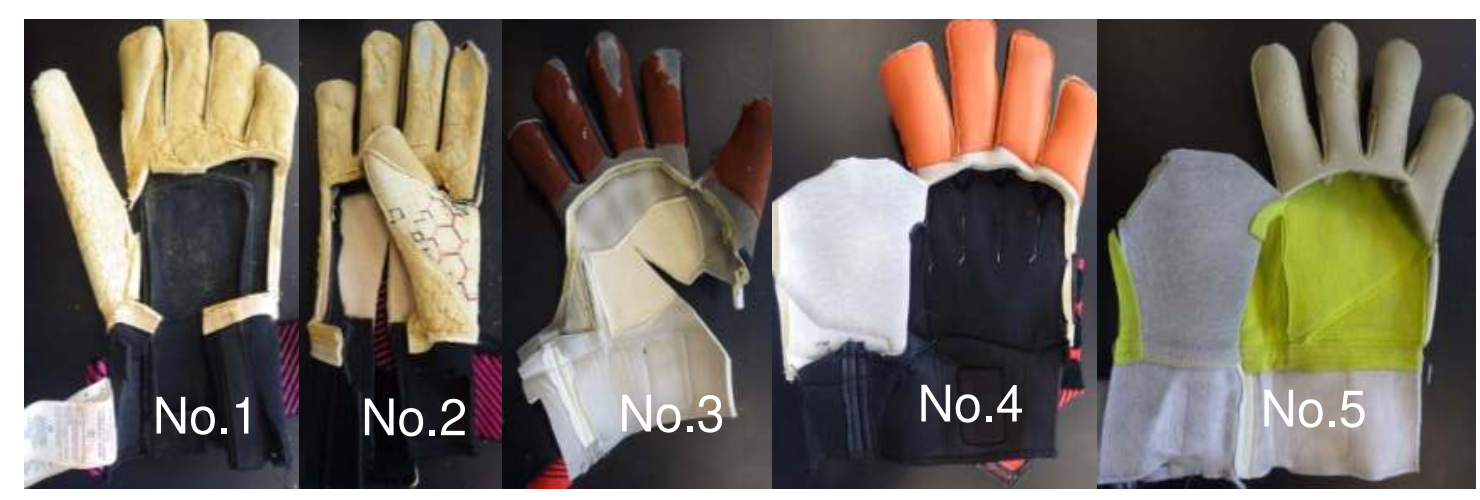

Figure 1. Clinical features and patient's 5 pairs of keeper gloves. (a)Pruritic erythema and fissures on the palms, dorsal hands and wrists. (b)We cut the 5 pairs of keeper gloves for PT.

\section{Patch Testing:}

We performed two-day closed PT with Japanese standard allergens using Finn chamber ${ }^{\circledR}$ on Scampor® tape or Patch test tape Torii ${ }^{\circledR}$, in addition to the patient's gloves. Readings were taken on Day2, 3 and 7 after application, according to the criteria of the International Contact Dermatitis Research Group. We judged totally that the positive reaction was positive after Day3.

\begin{tabular}{|c|c|}
\hline materials & D3 or D7 \\
\hline Gloves No.1 & + \\
\hline No.2 & + \\
\hline No.3 & + \\
\hline No.4 & + \\
\hline No.5 & + \\
\hline (patch test panel® $(\mathrm{S})$ ) & + \\
\hline PTBP-FR (Brial 1\%pet.) & + \\
\hline Carba mix $\quad$ patch test panel@ (S)) & + \\
\hline (Trolab 1\% pet.) & + \\
\hline
\end{tabular}

Table 1. Results of PT.

All 5 of the patient's gloves elicited a positive reaction. Among the Japanese standard allergens, $p$-tert- butylphenol-formaldehyde resin (PTBP-FR), Carba mix, and 1,3-diphenylguanidine (1,3-DPG), a component of Carba mix, also elicited a positive reaction. The others were negative.

\section{GC/MS:}

To confirm that patient's gloves include PTBP-FR and 1,3-DPG, NITE analyzed these gloves by GC/MS.
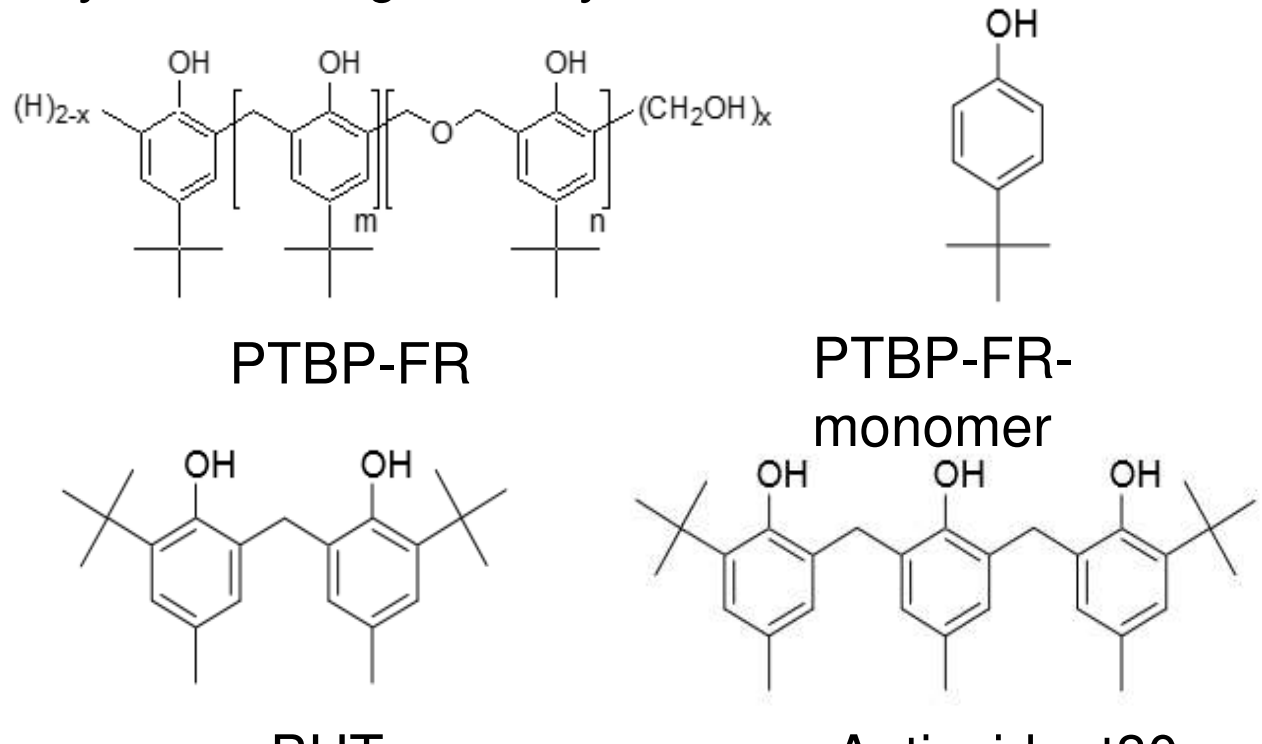

BKF

$\mathrm{BHT}$

Antioxidant80
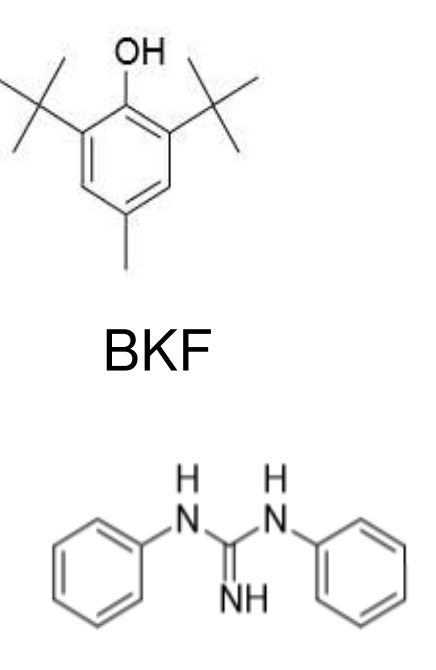

1,3-DPG

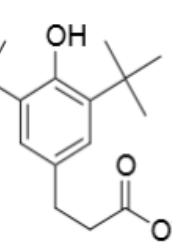

IRGANOX1076

Figure 2. Chemical structures in the patient's 5 gloves analyzed by GC/MS.

BHT , 2,6-Di-tert-butyl-p-cresol;

BKF , 2,2'-Methylenebis(6-tert-butyl-p-cresol);

Antioxidant80,2,6-Bis(3-tert-butyl-2-hydroxy-5-tolylmethyl)-4-methylphenol; IRGANOX1076, Octadecyl 3-(3,5-Di-tert-butyl-4-hydroxyphenyl)propionate;

\begin{tabular}{l|c|c|c|c|c|}
\multicolumn{1}{c|}{ materials } & \multicolumn{5}{c|}{ gloves } \\
\cline { 2 - 7 } & No.1 & No.2 & No.3 & No.4 & No.5 \\
\hline PTBP-FR monomer & + & + & - & + & - \\
\hline BHT & + & + & + & + & + \\
\hline BKF & + & + & + & + & + \\
\hline Antioxidant80 & + & + & + & - & + \\
\hline IRGANOX1076 & - & + & + & + & - \\
\hline 1,3-DPG & - & - & - & + & - \\
\hline
\end{tabular}

Table 2. Results of GC/MS in patient's 5 pairs of gloves

The ingredients with a similar structure to PTBP-FR were detectable in all of the patient's gloves, whereas 1,3-DPG was detectable in only one type of glove, No.4.

\section{Discussion:}

- PTBP-FR is a common component of glues in rubber products and 1,3DPG is used in rubber products as an accelerator. PTBP-FR consists of many substances, the majority of which are not chemically defined.

- In this case, PT demonstrated positive reaction to all 5 pairs of gloves, PTBP-FR and 1,3-DPG. We detected the ingredients with a similar structure to PTBP-FR were detectable in all of the patient's gloves, 1,3DPG was detectable in only one type of glove by GC/MS. We diagnosed him as having contact dermatitis due to PTBP-FR and 1,3-DPG.

- In general, it is extremely difficult to obtain information about ingredients in products from manufacturers. Therefore, in Japan, any skin disorders caused by daily life products that require treatment for 30 days or more are classified as "serious health damage" and NITE carries out the investigations, then inquiries can be made to the supplier.

\section{Conclusion:}

- We presented a case of contact dermatitis due to PRBP-FR and 1,3DPG.

- A system for chemical analysis of products like NITE is important to safely use daily life products.

\section{References:}

